\title{
GPS Assisted Location Based Service for Smart City with Cloud Integration
}

\author{
Rujuta Kharade ${ }^{1}$, Supriya Kulkarni ${ }^{1}$, Madhura Thorat ${ }^{1}$, Sneha Walhekar ${ }^{1}$ \\ Department of Computer Engineering, Pune, India ${ }^{1}$
}

\begin{abstract}
The potential ability of Global Positioning System (GPS) to assist navigating and tracking application facilitates in determining precise object positioning on earth. In order to efficiently execute tracking operation, GPS is dependent on various parameters. All the information gathered is then analyzed to accurately track the object in realtime. This paper deals with the comprehensive study of GPS, LBS and cloud integration. A location-based service (LBS) is a software level service that gives the precise information about the location to be tracked. In the proposed system the database is been stored on the cloud. The large database is been handled efficiently with the help of cloud integration techniques. The system uses various cloud services for the effective working for large database. Various queries are been evaluated for accessing the database. The user end implementations of location measurement processing algorithms are discussed. The real time position tracking system for the android applications are been used. Furthermore, the system will be proposed with end outcomes in the forms of graphs, text messages.
\end{abstract}

Keywords: Global Positioning System (GPS), Graphical user interfaces (GUI), Location Based Services (LBS).

\section{INTRODUCTION}

In daily routine mobile and information technology are integral part of our life.In this system an Application will be developed that makes Police department work easily and fast. System uses LBS and Data mining application in android. Police department can store the record files and manage huge amount of data efficiently. In this system, designing android application to overcome communication problem.During verification, collection and storing evidences of crime from this application is going to take less time for investigation.

\section{PROBLEM STATEMENT}

To implement Location Tracing and storing huge amount of Traffic related data that will provide functionality for solving problem such as, Updating and Manipulating of DatabaseVerifying the Records.

\section{SYSTEM DETAILS}

1. Android Application for the Traffic Police Men.

2. Web server .

3. GPS tracking for the android application.

4. Cloud for storing of large DataBase.

\section{REQUIREMENT ANALYSIS}

Some high value vehicles have advanced security systems fitted by the manufacturers already, which are nearly impossible to bypass. However, when the keys are stolen, all the systems fitted by the manufacturer offer absolutely no resistance to the thief in stealing the vehicle. There is a need to develop a system which can work independently of the car systems. When a vehicle is stolen, most of the time the owner realizes it only after it is too late. Hours pass between the actual theft and the knowledge of the theft reaching the owner and the police. With every passing minute the area of search gets bigger and bigger and the probability of the vehicle being found gets smaller.

Therefore, it would be useful to have a system which can alert the owner in realtime if the position of the vehicle is moved by a few meters. It would also be useful for parents to monitor the speeds at which their children drive to reduce the high accident risk posed by drivers under the age of 25 years, usually from over speeding. It would be useful to program an upper safe limit above which someone can be alerted. There is also a need for a system which can provide speed and location of the vehicle if and when needed. The cost factor and the simplicity of the system should also be considered. There should be no subscriptions, contracts, bonds or tie-ups which restrict the user to go to any other service 


\section{ISO 3297:2007 Certified}

Vol. 5, Issue 4, April 2017

provider. We propose a system to meet the above needs which has the ability to alert the owner in real time if the vehicle is moved a few meters from its parked location. The proposed design is cost-effective, reliable and has the function of accurate tracking.

\section{LITERATURE SURVEY}

\section{GPS Assisted Standard Positioning Service for Navigation and Tracking}

Keywords-GPS, Navigation, Tracking, modulation, frequency, satellite, SPS

Ability of Global Positioning System (GPS) to assist navigating and tracking application in determining object positioning on earth. It describes the implementation of the real time position tracking system which helps the them to locate the exact position of the specified system . In order to efficiently track objects on earth, the satellites in the constellation have to execute certain operations such as, C/A code generation, modulation, frequency planning etc. The location information is used along with survey maps and object control actuators to support navigation. This paper deals with the comprehensive study of GPS space segment and Control segment. Initially, the specification of GPS service known as Standard Positioning Service (SPS) ranging signal characteristics is introduced.

\section{Private and Precise Range Search for Location Based Services}

Keywords- Private, Precise, Trilateration, leverages, spatial cloaking, homomorphic encryption, POIs

This proposed system provides both privacy protection and result accuracy, for locations we required or which we have been specified. Trilateration Algorithm is been used to calculate the actual location. Trilateration algorithm calculates the three points of the specified location and with the help of these points it gives the extact location.This proposed system leverages the spatial cloaking technique used in previous methods, and complements it with homomorphic encryption for improving precision. One fundamental LBS is range search, which returns all Point of Interests (POIs) within a userspecified range. The returningresultsbyLBSareexactlythePOIswithinthespecified range, while LBS learns nothing about user's real location. LBS services raises increasing concern about location privacy. A number of techniques are proposed for $\mathrm{NN}$ search privacy protection.

\section{A real time GSM/GPS based tracking system based on GSM mobile phone}

Keywords-GSM, GPS, localization, alert, SMS

This paper presents a localization system, which is able to track the location and position with GPS and GSM devices. It also provides Alert messages to the system when the position of the agent is moving or not in defined position. We can get the current location of the System any time. A GPS device gets signals from two or more satellites to calculate its coordinates, which can be translated into geographical locations using online tools such as Google etc. The specifications concerning security and availability globally have led to implementations of passive systems that receive signals from a constellation of satellites. To exploit the near universal access to mobile phones and a global system for mobile communications (GSM), it is easy to develop an independent real-time tracking system that comprises of a GPS receiver, a microcontroller which processes the information from the GPS receiver and a GSM enabled modem which transmits and receives the information to and from a microcontroller. Real-time location alerts can be requested from the system and sent by it via the short message service (SMS).

\section{DeterminingPositionAroundanAsteroidUsing CommunicationRelaysandTrilateration}

Keywords-Asteroid, Relays, Trilateration, probes, spatial, orbiter

- These distance measurements in the proposed system, position can be determined using trilateration the same basic technique behind the earth-based GPS system.

- In this paper we consider the possibility of using a communicationssystemthatisoperatingbetweenprobesonthe surface of an asteroid and an orbiting satellite to determine their relative spatial positions.

- By measuring the round trip communication delay between the orbiter and various surface probes to estimate distance and, thus, the position.

5. Real-time tracking management system using GPS, GPRS and Google earth

Keywords-GPS, GPRS

- System includes GPS/ GPRS module to location acquisition and message transmition.

- Gives great stability and robust message transfer protocol that locations are accurately acquired and transmitted to server in real-time. 


\section{IJIREEICE \\ International Journal of Innovative Research in Electrical, Electronics, Instrumentation and Control Engineering \\ ISO 3297:2007 Certified \\ Vol. 5, Issue 4, April 2017}

\section{REAL TIME SURVEY}

- In previous years police used files to maintain all traffic records like word documents .

- Now a days, crime and corruption is increasing rapidly so maintaining records is going much difficult to handle, access and maintain.

- From this existing schedule we are designing purpose system to handle huge amount of data and manage it.

- It provides a common platform between police department, police and general public to share crime related information.

- We are also keeping a track on the traffic policemen.

\section{PROPOSED SYSTEM}

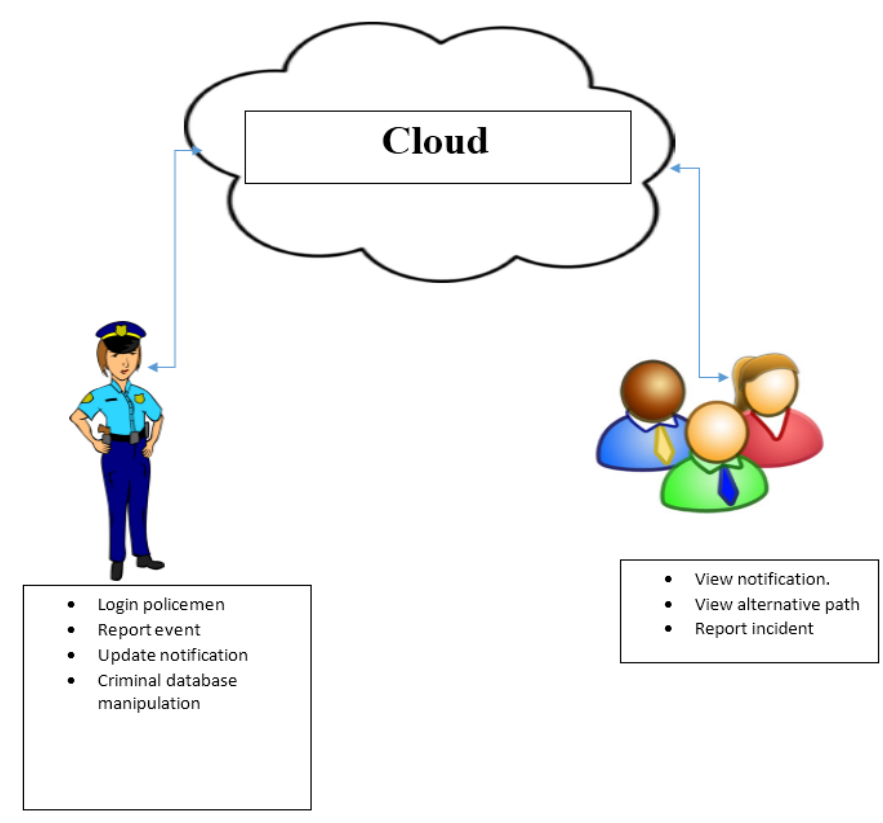

Fig: 1.1

\section{HOW IT WORKS}

Following are the screenshots of how our actual proposed system works :

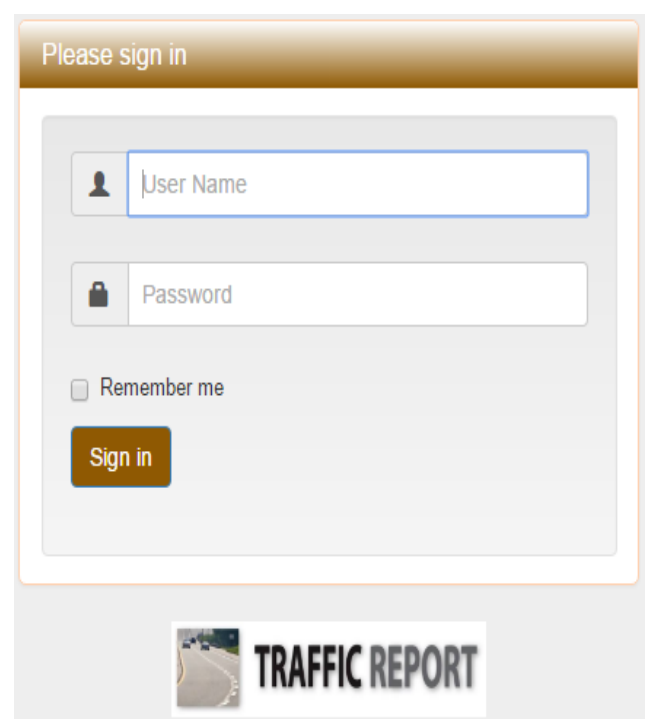

Fig. Login Page 


\section{International Journal of Innovative Research in Electrical, Electronics, Instrumentation and Control Engineering}

ISO 3297:2007 Certified

Vol. 5, Issue 4, April 2017

The administrator will login to this system.

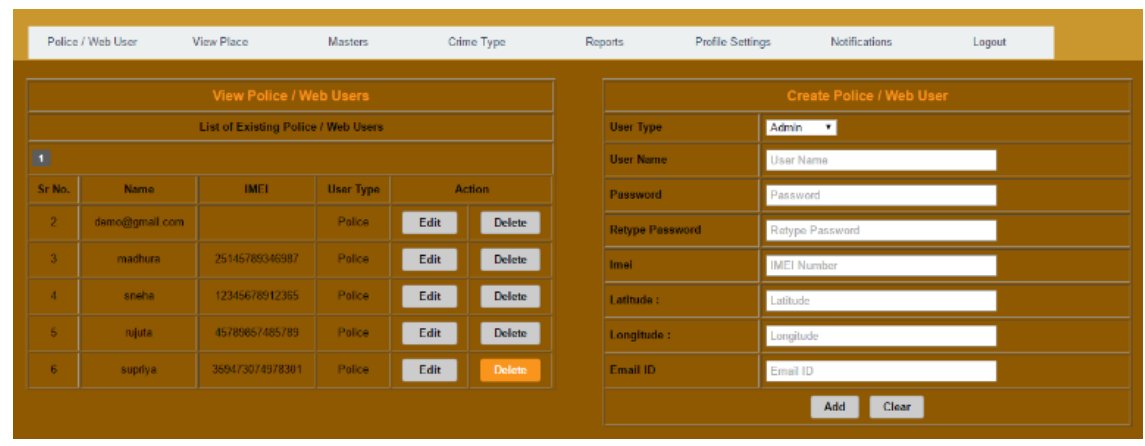

Fig. Web Portal

\section{CONCLUSION}

From this application we tried to overcome the problem of storing huge amount of information of traffic records , during investigation to retrieve and manage the data on cloud is solved from this android application and also overcome the problem of communication between police department, traffic police and user by our system.

\section{REFERENCES}

1. Afshan Mulla, Jaypal Baviskar†, Amol Baviskarł and Aniket Bhovad, Department of Electrical Engineering, Veermata Jijabai Technological Institute, Mumbai 400019, India."GPS Assisted Standard Positioning Service for Navigation and Tracking: Review \& Implementation” 2015 International Conference on Pervasive Computing (ICPC).

2. M. A. Al Rashed, Ousmane Abdoulaye Oumar, Damanjit Singh Faculty of Engineering, Science and the Built Environment, London South Bank University, London, UK "A real time GSM/GPS based tracking system based on GSM mobile phone "

3. Di Chen, Peng Zhang, Chengchen Hu, Huanzhao Wang*, Shun Wuł, and Ningzhe Xing Department of Computer Science and Technology, Xi' an Jiaotong University, Xi'an, China "Private and Precise Range Search for Location Based Services"IEEE ICC 2015

4. EvanNelson, Charles D. Creusere, Eric Butcher "Determining Position Around an Asteroid Using Communication Relays and Trilateration"

5. Chadil, N.Russameesawang, A.Keeratiwintakorn"Real-time tracking management system using GPS, GPRS and Google earth " 